October 22, 2018

\title{
CLUSTER EXPANSION IN THE CANONICAL ENSEMBLE
}

\author{
ELENA PULVIRENTI AND DIMITRIOS TSAGKAROGIANNIS
}

\begin{abstract}
We consider a system of particles confined in a box $\Lambda \subset \mathbb{R}^{d}$ interacting via a tempered and stable pair potential. We prove the validity of the cluster expansion for the canonical partition function in the high temperature - low density regime. The convergence is uniform in the volume and in the thermodynamic limit it reproduces Mayer's virial expansion providing an alternative and more direct derivation which avoids the deep combinatorial issues present in the original proof.
\end{abstract}

\section{INTRODUCTION}

The quantitative prediction of macroscopic properties of matter through its microscopic structure has been a main challenge for statistical mechanics. In this direction a very important theoretical as well as practical contribution is the work of J. E. and M. G. Mayer [13] (see also Ursell [19]) in the theory of non-ideal gases where they derive a full series expansion correcting the equation of state for the ideal gas $(p=k T \rho$, where $p$ is the pressure of the system at temperature $T$ with density $\rho, k$ being the Boltzmann constant) to all orders in $\rho$ obtaining the famous virial expansion. Convergence of this series has been addressed later (see [9] and [16]). The main idea in [13], (see also [12]) is to describe all possible interactions among the particles of the non-ideal gas by representing them as linear graphs, which has later led to a main tool in statistical mechanics, namely the cluster expansion method. The thermodynamic pressure is computed as the infinite volume limit of the logarithm of the grand canonical partition function, which is however a function of the activity of the system. To get an expansion with respect to the thermodynamic density, one needs to further express the latter in a power series of the activity, invert it and replace it in the equation for the pressure. This gives the virial expansion after using (for the inversion) some interesting combinatorial properties of enumeration of graphs which finally express the coefficients of the virial expansion to be sums over only "irreducible graphs".

This road which leads to an expansion of the free energy versus the density is evidently not the straight one! The direct and natural way is to take the density $\rho$ instead of the activity as order parameter and correspondingly to work in the canonical rather than in the grand canonical ensemble, which however rests on the possibility to cluster expand the canonical partition function. We came to this problem from other directions, see the end of the introduction, and found, to our surprise, that the problem is not only solvable but easy. It fits in fact beautifully in the theory of cluster expansion for abstract polymer models, as developed in all details by many authors after the pioneering work of [7], [6]. The exponentiation procedure in this theory produces a lot of diagrams which are not present in 
the Mayer expansion and which therefore must vanish in the thermodynamic limit. As we shall see in Section 5.1 the origin of such cancellations is closely related to the basic property of the cluster expansion (from which the expansion takes its name) namely that the only chains of graphs (clusters) which survive in the expansion are made of "incompatible" graphs. The validity of the cluster expansion for the canonical ensemble opens the way to attack several other problems (which was actually our initial motivation) such as the finite volume corrections to the free energy, the radius of convergence of the expansion in powers of the density (rather than the activity) for both the general model and the particularly interesting case of hard spheres, the construction of coarse-grained Hamiltonians via multi-canonical constraints as required by the Lebowitz-Penrose Theorem [10] for Kac interactions with applications to the LMP model [8] and its variants. We hope to further address these issues in subsequent papers.

\section{The MOdel AND The RESUlt}

We consider a configuration $\mathbf{q} \equiv\left\{q_{1}, \ldots, q_{N}\right\}$ of $N$ particles in a box $\Lambda:=\left(-\frac{L}{2}, \frac{L}{2}\right]^{d} \subset \mathbb{R}^{d}$ (for some $L>0$ ) which interact with a stable and tempered pair potential $V: \mathbb{R}^{d} \rightarrow \mathbb{R}$, i.e., there exists $B \geq 0$ such that:

$$
\sum_{1 \leq i<j \leq N} V\left(q_{i}-q_{j}\right) \geq-B N
$$

for all $N$ and all $q_{1}, \ldots, q_{N}$ and the integral

$$
C(\beta):=\int_{\mathbb{R}^{d}}\left|e^{-\beta V(q)}-1\right| d q
$$

is convergent for some $\beta>0$ (and hence for all $\beta>0$ ). Since in this paper we are interested in the infinite volume limit of the free energy density, we can assume periodic boundary conditions since it is a general result (see e.g. [16] and [5]) that the thermodynamic limit is independent of the choice of the boundary conditions. This particular choice in the present paper is not essential, periodic boundary conditions are used in order to obtain translation invariance in some cases (see e.g. Lemmas 4.1 and 5.2). Furthermore, our result remains valid with other boundary conditions by slightly changing the proof and we hope to address it in a subsequent work where we will consider the finite volume corrections as well.

We obtain the periodic boundary conditions by covering $\mathbb{R}^{d}$ with boxes $\Lambda$ and adding all interactions. Let

$$
V^{\text {per }}\left(q_{i}, q_{j}\right):=\sum_{n \in \mathbb{Z}^{d}} V\left(q_{i}-q_{j}+n L\right)
$$

where apart from assuming stability and temperedness, we further need to guarantee convergence of the above sum by imposing a condition on the decay properties of $V$. A potential $V$ is called lower regular if there exists a decreasing function $\psi: \mathbb{R}_{+} \rightarrow \mathbb{R}_{+}$such that $V(x) \geq-\psi(|x|)$ for all $x \in \mathbb{R}^{d}$ and $\int_{0}^{\infty} \psi(s) s^{d-1} d s<\infty$. Then $V$ will be called regular if it 
is lower regular and there exists some $r_{V}<\infty$ such that $V(x) \leq \psi(|x|)$ whenever $|x| \geq r_{V}$ and this is our extra assumption (see also the discussion in [5]).

The canonical partition function of the system with periodic boundary conditions is given by

$$
Z_{\beta, \Lambda, N}^{p e r}:=\frac{1}{N !} \int_{\Lambda^{N}} d q_{1} \ldots d q_{N} e^{-\beta H_{\Lambda}^{p e r}(\mathbf{q})},
$$

where $H_{\Lambda}^{\text {per }}$ is the energy of the system with periodic boundary conditions given by

$$
H_{\Lambda}^{\text {per }}(\mathbf{q}):=\sum_{1 \leq i<j \leq N} V^{\text {per }}\left(q_{i}, q_{j}\right)
$$

Given $\rho>0$ we define the thermodynamic free energy by

$$
f_{\beta}(\rho):=\lim _{|\Lambda|, N \rightarrow \infty, \frac{N}{|\Lambda|}=\rho} f_{\beta, \Lambda}(N), \text { where } f_{\beta, \Lambda}(N):=-\frac{1}{\beta|\Lambda|} \log Z_{\beta, \Lambda, N}^{\text {per }} .
$$

The main result in this paper is:

Theorem 2.1. If $\rho C(\beta)$ is small enough (see (4.6)) then

$$
\frac{1}{|\Lambda|} \log Z_{\beta, \Lambda, N}^{p e r}=\log \frac{|\Lambda|^{N}}{N !}+\frac{N}{|\Lambda|} \sum_{n \geq 1} F_{N, \Lambda}(n),
$$

where the coefficients $F_{N, \Lambda}(n), n \geq 1$, satisfy

$$
\left|F_{N, \Lambda}(n)\right| \leq C e^{-c n}
$$

for constants $C, c>0$ that are independent of $N$ and $\Lambda$. Moreover,

$$
F_{N, \Lambda}(n)=\frac{1}{n+1} P_{N,|\Lambda|}(n) B_{\beta, \Lambda}(n),
$$

where $P_{N,|\Lambda|}(n)$ and $B_{\beta, \Lambda}(n)$ are defined in (5.2) and which in the thermodynamic limit give

$$
\lim _{N,|\Lambda| \rightarrow \infty, N /|\Lambda|=\rho} P_{N,|\Lambda|}(n)=\rho^{n} \quad \text { and } \quad \lim _{\Lambda \rightarrow \infty} B_{\beta, \Lambda}(n)=\beta_{n},
$$

for all $n \geq 1$, where $\beta_{n}$ are the irreducible coefficients of Mayer:

$$
\beta_{n}:=\frac{1}{n !} \sum_{\substack{g \in \mathcal{B}_{n+1} \\ \text { supp } g \ni\{1\}}} \int_{\left(\mathbb{R}^{d}\right)^{n}} \prod_{\{i, j\} \in E(g)}\left(e^{-\beta V\left(q_{i}-q_{j}\right)}-1\right) d q_{2} \ldots d q_{n+1}, \quad q_{1} \equiv 0
$$

and $\mathcal{B}_{n+1}$ is the set of 2 -connected (or irreducible) graphs $g$ on $(n+1)$ vertices (i.e., connected graphs which by removing one vertex and all related edges remain connected) and $E(g)$ is the set of edges of the graph $g$.

Note the unfortunate coincidence of notation between the inverse temperature $\beta$ and the irreducible coefficients $\beta_{n}$, which however we keep in agreement with the literature. To prove Theorem 2.1 we first establish in Section 3 the set-up of the cluster expansion for the canonical partition function in the context of the abstract polymer model following [1], [14] and [7]. Note that we could also work with more general formulations such as in 
[15], [3] or [17]. In Section 4 we prove the convergence condition and as a corollary of the cluster expansion theorem we prove (2.8). The discussion of the thermodynamic limit is left for Section 5 where using the splitting in (2.9) we prove (2.10) by investigating the cancellations that lead to the "irreducible" coefficients $\beta_{n}$, given in (2.11). This latter fact is the result of some special structure (that we call "product structure") which may occur already in the abstract formulation of the polymer model and we address it in full generality in Subsection 5.1. Last, we also give an appendix with the main ideas of the original virial expansion and connect it to our approach. There is a number of interesting combinatorial issues that arise, many of which have been extensively studied in graph theory. With no intention of being exhaustive we just refer to [11] which is inspired by the virial expansion.

\section{Cluster expansion for the CANONicAl PARTition FUnCtion}

We view the canonical partition function $Z_{\beta, \Lambda, N}^{p e r}$ as a perturbation around the ideal case, hence normalizing the measure by multiplying and dividing by $|\Lambda|$ in (2.4) we write

$$
Z_{\beta, \Lambda, N}^{\text {per }}=Z_{\Lambda, N}^{\text {ideal }} Z_{\beta, \Lambda, N}^{\text {int }},
$$

where

$$
Z_{\Lambda, N}^{\text {ideal }}:=\frac{|\Lambda|^{N}}{N !} \quad \text { and } \quad Z_{\beta, \Lambda, N}^{\text {int }}:=\int_{\Lambda^{N}} \frac{d q_{1}}{|\Lambda|} \cdots \frac{d q_{N}}{|\Lambda|} e^{-\beta H_{\Lambda}^{\text {per }}(\mathbf{q})} .
$$

For $Z_{\beta, \Lambda, N}^{\text {int }}$ we use the idea of Mayer in [13] which consists of developing $e^{-\beta H_{\Lambda}^{p e r}(\mathbf{q})}$ in the following way

$$
e^{-\beta H_{\Lambda}^{\text {per }}(\mathbf{q})}=\prod_{1 \leq i<j \leq N}\left(1+f_{i, j}\right)=\sum_{g \in \mathcal{G}_{N}} \prod_{\{i, j\} \in E(g)} f_{i, j},
$$

where by $\mathcal{G}_{N}$ we denote the set of all simple graphs (undirected with no self-loops and no multiple edges) on $N$ vertices (the labels of the particles), where a graph is the pair $g \equiv$ $(V(g), E(g))$ where $V(g) \subset\{1, \ldots, N\}$ is the set of vertices and $E(g):=\{\{i, j\}, i, j \in V(g)\}$ is the set of (non-oriented) edges, and

$$
f_{i, j}:=e^{-\beta V^{p e r}\left(q_{i}-q_{j}\right)}-1 .
$$

Given a graph $g \in \mathcal{G}_{N}$ we say that it is connected if any two vertices of $g$ are connected by following its edges. We define the support of a graph (denoted by $\operatorname{supp} g$ ) to be the set of its vertices. Two graphs $g, g^{\prime}$ are compatible (denoted by $g \sim g^{\prime}$ ) if $\operatorname{supp} g \cap \operatorname{supp} g^{\prime}=\emptyset$ (i.e., they are not connected); otherwise we call them incompatible $(\nsim)$. We also denote by $|g|$ the cardinality of $\operatorname{supp} g$. With these definitions we see that any graph $g \in \mathcal{G}_{N}$ is equivalent to the pairwise compatible (non-ordered) collection of its connected components, i.e. $g \equiv\left\{g_{1}, \ldots, g_{k}\right\}_{\sim}$ for some $k$. Hence,

$$
Z_{\beta, \Lambda, N}^{\text {int }}:=\sum_{\left\{g_{1}, \ldots, g_{k}\right\} \sim} \prod_{i=1}^{k} \tilde{\zeta}_{\Lambda}\left(g_{i}\right)=\sum_{\left\{V_{1}, \ldots, V_{k}\right\} \sim} \prod_{i=1}^{k} \zeta_{\Lambda}\left(V_{i}\right),
$$


where

$$
\zeta_{\Lambda}(V):=\sum_{g \in \mathcal{C}_{V}} \tilde{\zeta}_{\Lambda}(g), \quad \tilde{\zeta}_{\Lambda}(g):=\int_{\Lambda|g|} \prod_{i \in \operatorname{supp} g} \frac{d q_{i}}{|\Lambda|} \prod_{\{i, j\} \in E(g)} f_{i, j} .
$$

Here $\mathcal{C}_{V}$ denotes the set of all connected graphs with support in $V \subset\{1, \ldots, N\}$. We will also be using the notation $\mathcal{C}_{n}$ for the set of all connected graphs on $n$ vertices. Both expressions in (3.5) are in the form of the abstract polymer model which we specify next.

An abstract polymer model $\left(\Gamma, \mathbb{G}_{\Gamma}, \omega\right)$ consists of (i) a set of polymers $\Gamma:=\left\{\gamma_{1}, \ldots, \gamma_{|\Gamma|}\right\}$, (ii) a binary symmetric relation $\sim$ of compatibility between the polymers (i.e., on $\Gamma \times \Gamma$ ) which is recorded into the compatibility graph $\mathbb{G}_{\Gamma}$ (the graph with vertex set $\Gamma$ and with an edge between two polymers $\gamma_{i}, \gamma_{j}$ if and only if they are an incompatible pair) and (iii) a weight function $\omega: \Gamma \rightarrow \mathbb{C}$. Then, we have the following formal relation which will become rigorous by Theorem 3.1 below (see [7], [1] and [14]):

$$
Z_{\Gamma, \omega}:=\sum_{\left\{\gamma_{1}, \ldots, \gamma_{n}\right\} \sim} \prod_{i=1}^{n} \omega\left(\gamma_{i}\right)=\exp \left\{\sum_{I \in \mathcal{I}} c_{I} \omega^{I}\right\}
$$

where

$$
c_{I}=\frac{1}{I !} \sum_{G \subset \mathcal{G}_{I}}(-1)^{|E(G)|}
$$

or equivalently $([1],[2])$

$$
c_{I}=\left.\frac{1}{I !} \frac{\partial^{\sum_{\gamma} I(\gamma)} \log Z_{\Gamma, \omega}}{\partial^{I\left(\gamma_{1}\right)} \omega\left(\gamma_{1}\right) \cdots \partial^{I\left(\gamma_{n}\right)} \omega\left(\gamma_{n}\right)}\right|_{\omega(\gamma)=0} .
$$

The sum in (3.7) is over the set $\mathcal{I}$ of all multi-indeces $I: \Gamma \rightarrow\{0,1, \ldots\}$ with $\operatorname{supp} I:=\{\gamma \in$ $\Gamma: I(\gamma)>0\}, \omega^{I}=\prod_{\gamma} \omega(\gamma)^{I(\gamma)}$ and $\mathcal{G}_{I}$ is the graph with $\sum_{\gamma \in \operatorname{supp} I} I(\gamma)$ vertices induced from $\mathcal{G}_{\text {supp } I} \subset \mathbb{G}_{\Gamma}$ by replacing each vertex $\gamma$ by the complete graph on $I(\gamma)$ vertices.

Furthermore, the sum in (3.8) is over all connected subgraphs $G$ of $\mathcal{G}_{I}$ spanning the whole set of vertices of $\mathcal{G}_{I}$ and $I !=\prod_{\gamma \in \operatorname{supp} I} I(\gamma)$ !. Note that if $I$ is supported on a compatible collection of polymers $\gamma$ then $c_{I}=0$.

We state the general theorem as in [1], [14] but in a slightly different form. Let

$$
L=L(\delta)=\sup _{x \in(0, \delta)}\left\{\frac{-\log (1-x)}{x}\right\}=\frac{-\log (1-\delta)}{\delta},
$$

where $\delta$ will be small and therefore $L=1+O(\delta)$. The optimal bound for the convergence radius is beyond the scope of the present paper, however, we hope to come back to this issue, also in the particular case of hard spheres, in a subsequent work.

Theorem 3.1. [Cluster Expansion] Assume that there are two non-negative functions a, $c$ : $\Gamma \rightarrow \mathbb{R}$ such that for any $\gamma \in \Gamma,|\omega(\gamma)| e^{a(\gamma)} \leq \delta$ holds, for some $\delta>0$ small. Moreover, 
assume that for any polymer $\gamma^{\prime}$

$$
\sum_{\gamma \nsim \gamma^{\prime}}|\omega(\gamma)| e^{a(\gamma)+c(\gamma)} \leq \frac{1}{L} a\left(\gamma^{\prime}\right),
$$

where $L$ is given in (3.10). Then, for any polymer $\gamma^{\prime} \in \Gamma$ the following bound holds

$$
\sum_{I: I\left(\gamma^{\prime}\right) \geq 1}\left|c_{I} \omega^{I}\right| e^{\sum_{\gamma \in \operatorname{supp} I} I(\gamma) c(\gamma)} \leq L\left|\omega\left(\gamma^{\prime}\right)\right| e^{a\left(\gamma^{\prime}\right)+c\left(\gamma^{\prime}\right)}
$$

where $c_{I}$ are given in (3.9).

Proof. Apply the proof in [1] for the activities $\omega(\gamma) e^{c(\gamma)}$.

In view of (3.5) we can represent the partition function $Z_{\beta, \Lambda, N}^{\text {int }}$ both as a polymer model on graphs with $\left(\mathcal{C}(N), \mathbb{G}_{\mathcal{C}}, \tilde{\zeta}_{\Lambda}\right)$ where we explicit the dependence on $N$ by denoting by $\mathcal{C}(N)$ the set of all labeled connected graphs on up to $N$ vertices and as a polymer model on vertices, with $\left(\mathcal{V}(N), \mathbb{G}_{\mathcal{V}}, \zeta_{\Lambda}\right)$ where $\mathcal{V}(N):=\{V: V \subset\{1, \ldots, N\}\}$.

\section{Convergence of the Cluster expansion, proof of (2.8)}

In this section we check the convergence condition of Theorem 3.1. We work in the case of vertices which corresponds to the abstract polymer model $\left(\mathcal{V}(N), \mathbb{G}_{\mathcal{V}}, \zeta_{\Lambda}\right)$ where $\mathcal{V}(N):=$ $\{V: V \subset\{1, \ldots, N\}\}$. Then, as a corollary of Theorem 3.1 we prove (2.8).

Lemma 4.1. There exist two positive functions $a, c: \mathcal{V}(N) \rightarrow \mathbb{R}$ such that for any $V \in$ $\mathcal{V}(N)$ :

$$
\left|\zeta_{\Lambda}(V)\right| e^{a(V)} \leq \delta
$$

holds for some $\delta>0$ small. Moreover, for any $V^{\prime} \in \mathcal{V}(N)$

$$
\sum_{V: V \nsim V^{\prime}}\left|\zeta_{\Lambda}(V)\right| e^{a(V)+c(V)} \leq \frac{1}{L} a\left(V^{\prime}\right)
$$

where $L$ is given in (3.10).

Proof. We choose $a(V)=a|V|$ and $c(V)=c|V|$, for some $a, c>0$ to be chosen later and let $\alpha:=a+c$. To bound $\left|\zeta_{\Lambda}(V)\right|$ we use a version of the tree-graph inequality (proved in this form in [15] and [16]) which states that for a stable and tempered potential, we have the following bound:

$$
\left|\sum_{g \in \mathcal{C}_{n}} \prod_{\{i, j\} \in E(g)} f_{i, j}\right| \leq e^{2 \beta B n} \sum_{T \in \mathcal{T}_{n}} \prod_{\{i, j\} \in E(T)}\left|f_{i, j}\right|,
$$


where $\mathcal{T}_{n} \subset \mathcal{C}_{n}$ is the set of trees with $n$ vertices. Then

$$
\begin{aligned}
\left|\zeta_{\Lambda}(V)\right| e^{a|V|} & \leq e^{(2 \beta B+a)|V|} \int_{\Lambda^{|V|}} \prod_{i \in V} \frac{d q_{i}}{|\Lambda|} \sum_{T \in \mathcal{T}_{|V|}} \prod_{\{i, j\} \in E(T)}\left|f_{i, j}\right| \\
& \leq e^{(2 \beta B+a) n} \sum_{T \in \mathcal{T}_{n}} \int_{\Lambda^{n}} \frac{d q_{1}}{|\Lambda|} \cdots \frac{d q_{n}}{|\Lambda|} \prod_{\{i, j\} \in E(T)}\left|f_{i, j}\right| .
\end{aligned}
$$

Given a rooted tree $T$ let us call $\left(i_{1}, j_{1}\right),\left(i_{2}, j_{2}\right), \ldots,\left(i_{n-1}, j_{n-1}\right)$ its edges. We have:

$$
\begin{aligned}
\int_{\Lambda^{n}} \frac{d q_{1}}{|\Lambda|} \cdots \frac{d q_{n}}{|\Lambda|} \prod_{\{i, j\} \in E(T)}\left|f_{i, j}\right| & =\frac{1}{|\Lambda|^{n}} \int_{\Lambda^{n}} d q_{1} \cdots d q_{n} \prod_{k=1}^{n-1}\left|f_{i_{k}, j_{k}}\right| \\
& \leq \frac{1}{|\Lambda|^{n}} \int_{\Lambda} d q_{i_{1}} \int_{\Lambda} d y_{2} \cdots \int_{\Lambda} d y_{n} \prod_{k=2}^{n}\left|e^{-\beta V^{p e r}\left(y_{k}\right)}-1\right| \\
& \leq \frac{|\Lambda|}{|\Lambda|^{n}}\left[\int_{\Lambda} d x\left|e^{-\beta V^{p e r}(x)}-1\right|\right]^{n-1}=: \frac{|\Lambda|}{|\Lambda|^{n}} C_{\Lambda}(\beta)^{n-1}
\end{aligned}
$$

(note that the choice of $V^{\text {per }}$ makes $C_{\Lambda}(\beta)$ independent of $x$ ) where we considered $q_{i_{1}}$ as the root and we used the change of variables:

$$
y_{k}=q_{i_{k}}-q_{j_{k}}, \quad \forall k=2, \ldots, n .
$$

We choose $\rho C(\beta)$ such that:

$$
\rho e^{2 \beta B+\alpha+1} C(\beta)<1 .
$$

Then, since the number of all trees in $\mathcal{T}_{n}$ is $n^{n-2}$, from (4.4) we obtain (recalling that $N /|\Lambda|=\rho)$ :

$$
\left|\zeta_{\Lambda}(V)\right| e^{a|V|} \leq \frac{n^{n-2}}{|\Lambda|^{n-1}} e^{(2 \beta B+a) n} C_{\Lambda}(\beta)^{n-1} \leq \frac{1}{2} \rho C_{\Lambda}(\beta) e^{2(2 \beta B+a)},
$$

by using the bound $2 \leq n \leq N$ and the fact that $\rho e^{2 \beta B+a} C_{\Lambda}(\beta)<1$. The latter is true considering that inequality (4.6) still holds with $C_{\Lambda}(\beta)$ for $\Lambda$ large enough, since $\lim _{\Lambda \rightarrow \infty} C_{\Lambda}(\beta)=C(\beta)$. Then defining $\delta:=\frac{1}{2} \rho C(\beta) e^{2(2 \beta B+a)}$, (4.1) is satisfied. Considering $a=1$ we have (for any fixed $i$ ):

$$
\sum_{V: V \ni i}\left|\zeta_{\Lambda}(V)\right| e^{\alpha|V|} \leq \sum_{n \geq 2}\left(\begin{array}{c}
N-1 \\
n-1
\end{array}\right) \frac{n^{n-2}}{|\Lambda|^{n-1}} e^{(2 \beta B+\alpha) n} C_{\Lambda}(\beta)^{n-1} \leq e \delta \frac{1}{1-\delta^{\prime}} \leq \frac{1}{L},
$$

recalling that $L=1+O(\delta)$ and where $\delta^{\prime}:=\rho e^{2 \beta B+\alpha+1} C(\beta)$. Since $\left\{V \not V^{\prime}\right\} \subset \bigcup_{i \in V^{\prime}}\{V \ni i\}$ we get (4.2) and conclude the proof of the lemma.

The way we chose to present the cluster expansion as well as its convergence can by no means give the best radius of convergence. Our goal was merely to obtain (giving up the seek for the best radius) the consequence of the cluster expansion theorem, given in (3.12), which we use in order to establish (2.7). Nevertheless, our condition is comparable with the ones in 
the literature (see [9], equation (3.15), and in [16], Theorem 4.3.2) and we will clarify these issues in a subsequent work. Moreover, for the particular case of the hard spheres we can obtain the improved radius as in [4] but for the density $\rho$ rather than the activity, since we are working directly with the canonical partition function.

Proof of (2.8). After proving the convergence condition it follows directly from Theorem 3.1 that for all $V^{\prime} \in \mathcal{V}(N)$ and by choosing $c(V):=c|V|$ and $a(V):=|V|$

$$
\sum_{I: I\left(V^{\prime}\right) \geq 1}\left|c_{I} \zeta_{\Lambda}^{I}\right| e^{c\|I\|} \leq L\left|\zeta_{\Lambda}\left(V^{\prime}\right)\right| e^{\alpha\left|V^{\prime}\right|}, \quad\|I\|:=\sum_{V \in \operatorname{supp} I} I(V)|V|
$$

where we remind that $\alpha=1+c$. Thus, exponentiating the partition function (3.5) using (3.7), we obtain $(2.7)$ :

$$
\frac{1}{|\Lambda|} \log Z_{\beta, \Lambda, N}^{i n t}=\frac{1}{|\Lambda|} \sum_{I} c_{I} \zeta_{\Lambda}^{I}=\frac{1}{|\Lambda|} \sum_{i \in\{1, \ldots, N\}} \sum_{I: \operatorname{supp} I \ni\{i\}} \frac{1}{|I|} c_{I} \zeta_{\Lambda}^{I}=\frac{N}{|\Lambda|} \sum_{n \geq 1} F_{N, \Lambda}(n),
$$

where

$$
F_{N, \Lambda}(n):=\frac{1}{n+1} \sum_{\substack{I: \operatorname{supp} I \ni\{1\} \\|I|=n+1}} c_{I} \zeta_{\Lambda}^{I}, \quad|I|:=\left|\bigcup_{V \in \operatorname{supp} I} V\right|
$$

Note that $|I| \leq\|I\|$. The function $F_{N, \Lambda}(n)$ is uniformly bounded for all $N, \Lambda$ as well as absolutely summable over $n$, namely from (4.9) with $V^{\prime} \equiv\{1\}$ we get:

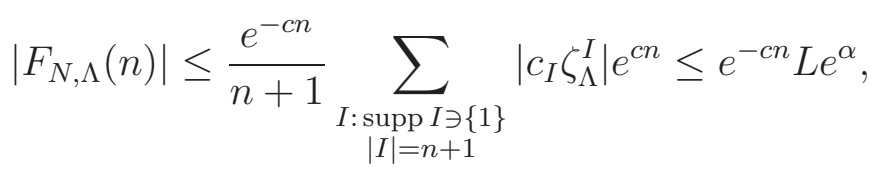

which concludes the proof of (2.8).

Note that the sum in (4.11) is infinite. As it will be apparent in the next section it is more convenient to work with a truncated version of $F_{N, \Lambda}(n)$. Thus, for $M \geq n+1$ we define

$$
F_{N, \Lambda}^{M}(n):=\frac{1}{n+1} \sum_{\substack{I: \operatorname{supp} I \ni\{1\} \\|I|=n+1,\|I\| \leq M}} c_{I} \zeta_{\Lambda}^{I}
$$

which is uniformly bounded for all $N, \Lambda$ and absolutely summable over $n$ using the same argument as in (4.12). Note also that now the sum is finite. Furthermore, for all $n \geq 1$

$$
\sup _{N, \Lambda}\left|F_{N, \Lambda}^{M}(n)-F_{N, \Lambda}(n)\right| \leq \sup _{N, \Lambda} \frac{e^{-\frac{c}{2}(n+M)}}{n+1} \sum_{\substack{I: \operatorname{supp} I \ni\{1\} \\|I|=n+1,\|I\|>M}}\left|c_{I} \zeta_{\Lambda}^{I}\right| e^{\frac{c}{2}(n+M)} \leq e^{-\frac{c}{2}(n+M)} L e^{\alpha},
$$

since inside the sum we have: $n \leq M<\|I\|$. Hence, we can next work with the truncated function $F_{N, \Lambda}^{M}(n)$ and then pass simultaneously to both limits: the thermodynamic and $M \rightarrow \infty$. 
5. The thermodynamic Limit, PRoOF of (2.10)

The sum in the definition of $F_{N, \Lambda}^{M}(n)$ (4.13) does not depend on the labels of the extra $n$ particles (we have already chosen label 1). Hence,

$$
F_{N, \Lambda}^{M}(n)=\frac{1}{n+1}\left(\begin{array}{c}
N-1 \\
n
\end{array}\right) \sum_{\substack{I:\|I\| \leq M \\
\operatorname{supp} I \equiv\{1, \ldots, n+1\}}} c_{I} \zeta_{\Lambda}^{I}=\frac{1}{n+1} P_{N,|\Lambda|}(n) B_{\beta, \Lambda}^{M}(n),
$$

where

$$
P_{N,|\Lambda|}(n):=\frac{(N-1) \ldots(N-n)}{|\Lambda|^{n}} \quad \text { and } \quad B_{\beta, \Lambda}^{M}(n):=\frac{|\Lambda|^{n}}{n !} \sum_{\substack{I:\|I\| \leq M \\ \operatorname{supp} I \equiv\{1, \ldots, n+1\}}} c_{I} \zeta_{\Lambda}^{I} .
$$

While obviously $P_{N,|\Lambda|}(n) \rightarrow \rho^{n}$, for $B_{\beta, \Lambda}^{M}(n)$ we proceed as follows: we have restricted ourselves to a system of only $n+1$ particles, therefore we can view the sum in $B_{\beta, \Lambda}^{M}(n)$ as the cluster sum in $\left(\mathcal{V}(n+1), \mathbb{G}_{\mathcal{V}}, \zeta_{\Lambda}\right)$ with the extra condition $\|I\| \leq M$. While for the convergence it was more convenient to work with the space $\left(\mathcal{V}(N), \mathbb{G} \mathcal{V}, \zeta_{\Lambda}\right)$, the cancellations that we discuss in this section occur already at the more elementary structure of labeled connected graphs (i.e., on $\left(\mathcal{C}(n+1), \mathbb{G}_{\mathcal{C}}, \tilde{\zeta}_{\Lambda}\right)$ whose multi-indeces we denote by $\tilde{I}: \mathcal{C}(n+$ $1) \rightarrow\{0,1, \ldots\})$ so since $\sum_{I:\|I\| \leq M} c_{I} \zeta_{\Lambda}^{I}=\sum_{\tilde{I}:\|\tilde{I}\| \leq M} c_{\tilde{I}} \tilde{\zeta}_{\Lambda}^{\tilde{I}}$ (coming from the same partition function) we rewrite $B_{\beta, \Lambda}^{M}(n)$ as follows:

$$
B_{\beta, \Lambda}^{M}(n)=\frac{|\Lambda|^{n}}{n !} \sum_{\tilde{I}:\|\tilde{I}\| \leq M} c_{\tilde{I}} \tilde{\zeta}_{\Lambda}^{\tilde{I}}=\frac{|\Lambda|^{n}}{n !} \sum_{g \in \mathcal{C}_{n+1}} \sum_{\substack { \tilde{I}:\|\tilde{I}\| \leq M \\
\begin{subarray}{c}{\mathfrak{g}^{\prime} \in \operatorname{supp} \tilde{I} g^{\prime}=g{ \tilde { I } : \| \tilde { I } \| \leq M \\
\begin{subarray} { c } { \mathfrak { g } ^ { \prime } \in \operatorname { s u p p } \tilde { I } g ^ { \prime } = g } }\end{subarray}} c_{\tilde{I}} \tilde{\zeta}_{\Lambda}^{\tilde{I}}
$$

Note that looking at the support of some cluster $\tilde{I}$ we see a connected graph (with possible superposition of some graphs $g$ if $\tilde{I}(g) \geq 2$, or with some common parts of a graph if they are incompatible in more than one points). Thus, in (5.3) we first put in evidence the resulting graph $g \in \mathcal{C}_{n+1}$ and then sum over all $\tilde{I}$ that correspond to it. The main idea of what follows is to investigate the order in $|\Lambda|$ of the products $\tilde{\zeta}_{\Lambda}^{\tilde{I}}$. We start with a definition:

Definition 5.1. Given a connected undirected graph a vertex is said to be an articulation point if removing it and all edges incident to it the graph results in a non-connected graph.

One of the main ingredients of our proof is the fact that if $\left\{g^{\prime}: g^{\prime} \in \operatorname{supp} \tilde{I}\right\}$ are connected at only articulation points of the fixed $g \in \mathcal{C}_{n+1}$ then the activities $\tilde{\zeta}_{\Lambda}$ factorize. To emphasize this fact we state it as a lemma:

Lemma 5.2. Given $g \in \mathcal{C}_{n+1}$ and an incompatible collection $G$ such that $\cup_{g^{\prime} \in G} g^{\prime}=g$, if for all incompatible pairs $g^{\prime}, g^{\prime \prime}$ we have that $\operatorname{supp} g^{\prime} \cap \operatorname{supp} g^{\prime \prime}=\{i\}$ is a singleton and moreover, the vertex $i$ is an articulation point of $g$ then

$$
\tilde{\zeta}_{\Lambda}(g)=\prod_{g^{\prime} \in G} \tilde{\zeta}_{\Lambda}\left(g^{\prime}\right)
$$


for all finite $\Lambda$.

Proof. By the definition of an articulation point there is a $g^{\prime} \in G$ which has only one articulation point. We integrate the coordinates in $g^{\prime}$ keeping fixed the one that corresponds to the articulation point. In principle the result should depend on the position of the articulation point, but this is ruled out by the special choice of boundary conditions (as we stressed in Section 2) which makes such integrals translation invariant. Hence, the result of the integration gives $\tilde{\zeta}_{\Lambda}\left(g^{\prime}\right)$. Then, again by the definition of an articulation point, this can continue until integrating all $g^{\prime} \in G$.

Coming back to (5.3) the power of $|\Lambda|$ is $n-\sum_{g^{\prime} \in \operatorname{supp} \tilde{I}}\left(\left|g^{\prime}\right|-1\right) \tilde{I}\left(g^{\prime}\right)$. Moreover, since it is always true that $n+1 \leq \sum_{g^{\prime} \in \operatorname{supp} \tilde{I}}\left(\left|g^{\prime}\right|-1\right)+1$ (by the fact that all $g^{\prime} \in \operatorname{supp} \tilde{I}$ should be incompatible, i.e., have at least one common label) it is implied that non-negligible terms (in the limit $\Lambda \rightarrow \infty$ ) should satisfy

$$
\begin{aligned}
& \tilde{I}\left(g^{\prime}\right)=1, \forall g^{\prime} \in \operatorname{supp} \tilde{I}, \text { and } \\
& n+1=\sum_{g^{\prime} \in \operatorname{supp} \tilde{I}}\left(\left|g^{\prime}\right|-1\right)+1 .
\end{aligned}
$$

(In the representation of hyper-graphs by bi-partite graphs, these two requirements correspond to graphs without loops.) Again, to emphasize we state it as a lemma

Lemma 5.3. Given $\tilde{I}$, if there is $A \subset \operatorname{supp} \tilde{I}$ such that $\tilde{\zeta}_{\Lambda}\left(\cup_{g^{\prime} \in A} g^{\prime}\right) \neq \prod_{g^{\prime} \in A} \tilde{\zeta}_{\Lambda}\left(g^{\prime}\right)$ then the cluster that corresponds to $\tilde{I}$ is vanishing at the thermodynamic limit.

Proof. Suppose that there is such a $A \subset \tilde{I}$, i.e.,

$$
\left|\bigcup_{g^{\prime} \in A} g^{\prime}\right| \neq \sum_{g^{\prime} \in A}\left(\left|g^{\prime}\right|-1\right)+1
$$

Then, (5.7) implies that

$n+1=\left|\bigcup_{g^{\prime} \in \operatorname{supp} \tilde{I}} g^{\prime}\right| \leq\left|\bigcup_{g^{\prime} \in A} g^{\prime}\right|+\left|\bigcup_{g^{\prime} \in \operatorname{supp} \tilde{I} \backslash A} g^{\prime}\right|-1<\sum_{g^{\prime} \in A}\left(\left|g^{\prime}\right|-1\right)+1+\sum_{g^{\prime} \in \operatorname{supp} \tilde{I} \backslash A}\left(\left|g^{\prime}\right|-1\right)+1-1$,

hence (5.6) cannot be true and it has to vanish by taking the limit.

Proof of (2.10). Motivated by these facts we proceed with (5.3) by splitting the sum over $\tilde{I}$ into the following two complementary cases:

(1) (factorization property holds) $\tilde{\zeta}_{\Lambda}\left(\cup_{g \in A} g\right)=\prod_{g \in A} \tilde{\zeta}_{\Lambda}(g)$ for all $A \subset \operatorname{supp} \tilde{I}$,

(2) there exists $A \subset \operatorname{supp} \tilde{I}$ such that $\tilde{\zeta}_{\Lambda}\left(\cup_{g \in A} g\right) \neq \prod_{g \in A} \tilde{\zeta}_{\Lambda}(g)$.

Lemma 5.3 states that in the limit $|\Lambda| \rightarrow \infty$ the terms of Case (2) vanish (and they are finitely many due to the truncation). For the case (1), if $g \in \mathcal{C}_{n+1} \backslash \mathcal{B}_{n+1}$ then all terms in 
$B_{\beta, \Lambda}^{M}(n)$ are exactly canceling each other. This is a property of the combinatorial coefficients multiplying products of activities of polymers which have the factorization property, just as in the cluster expansion where compatible collections (carrying the factorization property) do not contribute in the sum. In the next subsection we investigate this property that we call "product structure" (see Definition 5.1) and since it can be a general property of the abstract polymer model we prove it in its general form in Lemma 5.5 and then we explicit it in our case in Corollary 5.6.

Thus, the only remaining terms are those of Case 1 for $g \in \mathcal{B}_{n+1}$ which due to condition (5.5) give non-vanishing contribution only for $\tilde{I}(g)=1$ (and those with higher multiplicity are only finitely many, due to the truncation). Hence, for all $n \geq 1$ we can pass to the thermodynamic limit (note that (4.14) permits to exchange sum and limits) and obtain:

$$
\lim _{\Lambda \rightarrow \infty} B_{\beta, \Lambda}(n)=\lim _{\Lambda \rightarrow \infty} \lim _{M \rightarrow \infty} B_{\beta, \Lambda}^{M}(n)=\lim _{\Lambda \rightarrow \infty} \frac{|\Lambda|^{n}}{n !} \sum_{g \in \mathcal{B}_{n+1}} \tilde{\zeta}_{\Lambda}(g)=\beta_{n},
$$

where $\beta_{n}$ is defined in (2.11), concluding (2.10) and the proof of Theorem 2.1 (pending the proof of Corollary 5.6).

5.1. The "product structure". Coming back to the general formulation $\left(\Gamma, \mathbb{G}_{\Gamma}, \omega\right)$, for any $\Gamma^{\prime} \subset \Gamma$ we define the set of all incompatible sequences that can be constructed out of elements of $\Gamma^{\prime}$ by:

$$
\Gamma_{\nsim}^{\prime}:=\left\{A: A \subset \Gamma^{\prime}, \text { incompatible }\right\} .
$$

Recall that every single element $\gamma \in \Gamma^{\prime}$ is considered incompatible, hence the singleton $\{\gamma\}$ is an element in $\left.\Gamma_{\nsim}^{\prime}\right)$.

Definition 5.4. Given $\left(\Gamma, \mathbb{G}_{\Gamma}, \omega\right)$, we say that $\Gamma^{b} \subset \Gamma$ has a "product structure" if

- there exists a one-to-one function $\phi: \Gamma_{\nsim}^{b} \rightarrow \Gamma$, with $\phi(\{\gamma\})=\gamma$, if $\gamma \in \Gamma^{b}$,

- for any $A \in \Gamma_{\nsim}^{b}$, we have the factorization

$$
\omega(\phi(A))=\prod_{\gamma^{\prime} \in A} \omega\left(\gamma^{\prime}\right) .
$$

We also define the range of $\phi$ by

$$
\mathrm{R}(\phi):=\left\{\phi(A), \forall A \in \Gamma_{\nsim}^{b}\right\} .
$$

We are interested in all multi-indeces $I$ such that every $\gamma \in \operatorname{supp} I$ is the image of some $A \in \Gamma_{\nsim}^{b}$, i.e., supp $I \subset \mathrm{R}(\phi)$ and this is the content of the following lemma.

Lemma 5.5. If $\Gamma^{b} \subset \Gamma$ has a product structure then in the expansion (3.7) we have:

$$
\sum_{I: \operatorname{supp} I \subset \mathrm{R}(\phi)} c_{I} \omega^{I}=\sum_{I: \operatorname{supp} I \equiv\left\{\gamma^{\prime}\right\}, \gamma^{\prime} \in \Gamma^{b}} c_{I} \omega^{I} .
$$


Proof. Given $\Gamma^{b} \subset \Gamma$ with product structure, using (5.12), we define

$$
\begin{aligned}
Z^{*}\left(\Gamma^{b}\right) & :=Z_{\Gamma,\{\omega(\gamma) \equiv 0, \forall \gamma \notin \mathrm{R}(\phi)\}} \equiv \sum_{\substack{\left\{\gamma_{1}, \ldots, \gamma_{k}\right\} \sim \\
\gamma_{i} \in \mathrm{R}(\phi)}} \prod_{i=1}^{k} \omega\left(\gamma_{i}\right) \\
& =\sum_{\substack{\left\{A_{1}, \ldots, A_{k}\right\} \sim \\
\phi\left(A_{i}\right)=\gamma_{i}, \forall i}} \prod_{i=1}^{k} \omega\left(\phi\left(A_{i}\right)\right)=\prod_{\gamma^{\prime} \in \Gamma^{b}}\left(1+\omega\left(\gamma^{\prime}\right)\right) .
\end{aligned}
$$

The first equality of (5.14) is due to the fact that $\phi$ is one-to-one, i.e., for any $\gamma_{i} \in \mathrm{R}(\phi)$ there is a unique $A_{i} \in \Gamma_{\nsim}^{b}$ with $\phi\left(A_{i}\right)=\gamma_{i}$. Then using the factorization property, i.e., $\omega\left(\phi\left(A_{i}\right)\right)=\prod_{\gamma^{\prime} \in A_{i}} \omega\left(\gamma^{\prime}\right)$, the second equality is due to the fact that $\prod_{\gamma^{\prime} \in \Gamma^{b}}\left(1+\omega\left(\gamma^{\prime}\right)\right)=$ $\sum_{A \subset \Gamma^{b}} \prod_{\gamma^{\prime} \in A} \omega\left(\gamma^{\prime}\right)$, where the latter sum is over subsets $A$ (compatible or incompatible) of the set of vertices in $\Gamma^{b}$, hence it can be uniquely decomposed into $k$ compatible components $A \equiv\left\{A_{1}, \ldots, A_{k}\right\}_{\sim}$ with $A_{i} \in \Gamma_{\nsim}^{b}$ for all $i$.

Then if we exponentiate the last expression of (5.14) we obtain the right hand side of (5.13), while if we exponentiate $Z_{\Gamma,\{\omega(\gamma) \equiv 0, \forall \gamma \notin \mathrm{R}(\phi)\}}$ (by first exponentiating using (3.9) and then evaluating) we obtain the left hand side of (5.13).

Now we explicit the result of Lemma 5.5 for the case of (5.3), which corresponds to the particular polymer model $\left(\mathcal{C}(n+1), \mathbb{G}_{\mathcal{C}}, \tilde{\zeta}_{\Lambda}\right)$. Any $g \in \mathcal{C}_{n+1} \backslash \mathcal{B}_{n+1}$ can be uniquely decomposed into 2-connected components, say $g=\cup_{i=1}^{k} b_{i}$ where $b_{i} \in \mathcal{B}(n+1), \forall i=1, \ldots, k$ (recall the difference of notation between $\mathcal{B}_{n+1}$ and $\mathcal{B}(n+1)$ with the latter being the set of 2connected graphs on up to $n+1$ vertices). Then, according to Definition 5.4 , any such element $\left\{b_{1}, \ldots, b_{k}\right\}$ has product structure with $\phi(A):=\cup_{b \in A} b$ for all $A \subset\left\{b_{1}, \ldots, b_{k}\right\}$ incompatible and it also satisfies (see Lemma 5.2) the factorization property by construction. Thus,

Corollary 5.6. Given $g \in \mathcal{C}_{n+1} \backslash \mathcal{B}_{n+1}$, then

$$
\sum_{\substack{\tilde{I}:\|\tilde{I}\| \leq M \\ \cup}}^{*} c_{g^{\prime} \in \operatorname{supp}} \tilde{\zeta}_{g^{\prime}=g}^{\tilde{I}}=0,
$$

for all truncations $M$ and where $*$ is to remind that the sum is over $\tilde{I}$ who are supported on polymers $g$ which have the factorization property, as in Case 1 in the proof of (2.10).

Proof. Suppose that $\left\{b_{1}, \ldots, b_{k}\right\}$ is the unique decomposition into 2-connected components of $g$ and hence has product structure. Then applying Lemma 5.5 for $\Gamma^{b}:=\left\{b_{1}, \ldots, b_{k}\right\}$ we obtain that all coefficients of $\tilde{\zeta}_{\Lambda}\left(b_{1}\right)^{n_{1}} \ldots \tilde{\zeta}_{\Lambda}\left(b_{k}\right)^{n_{k}}$ (with $n_{1}\left|b_{1}\right|+\ldots+n_{k}\left|b_{k}\right| \leq M$ ) are zero except those with $k=1$, which however correspond to the case $g=b_{1} \in \mathcal{B}_{n+1}$ and are not considered since we assumed $g \in \mathcal{C}_{n+1} \backslash \mathcal{B}_{n+1}$. 


\section{APPEndix: MAYER's VIRIAl EXPANSION}

The approach introduced by Mayer, see [13], is to work in the grand canonical ensemble which is a measure on both the number of particles $N$ and the configuration $\mathbf{q}$

$$
G_{\beta, z, \Lambda}(N ; d \mathbf{q}):=\frac{1}{\Xi_{\beta, \Lambda}(z)} z^{N} e^{-\beta H_{\Lambda}(\mathbf{q})} d q_{1} \ldots d q_{N},
$$

where $\Xi_{\beta, \Lambda}(z)$ is the grand canonical partition function given by

$$
\Xi_{\beta, \Lambda}(z):=\sum_{N \geq 0} z^{N} Z_{\beta, \Lambda, N}
$$

and $z$ is the activity of the system. The thermodynamic pressure is defined as the infinite volume limit of the logarithm of the grand canonical partition function:

$$
p_{\beta}(z):=\lim _{|\Lambda| \rightarrow \infty} p_{\beta, \Lambda}(z), \quad \text { where } \beta p_{\beta, \Lambda}(z)=\frac{1}{|\Lambda|} \log \Xi_{\beta, \Lambda}(z) .
$$

The idea in [13] consists of developing $e^{-\beta H_{\Lambda}(\mathbf{q})}$ in the following way

$$
e^{-\beta H_{\Lambda}(\mathbf{q})}=\prod_{1 \leq i<j \leq N}\left(1+f_{i, j}\right)=\sum_{g \in \mathcal{G}_{N}} \prod_{\{i, j\} \in E(g)} f_{i, j},
$$

where by $\mathcal{G}_{N}$ we denote all simple graphs on $N$ vertices, $E(g)$ is the set of edges of a graph $g \in \mathcal{G}_{N}$ and

$$
f_{i, j}:=e^{-\beta V\left(q_{i}-q_{j}\right)}-1 .
$$

Then the grand canonical partition function becomes

$$
\Xi_{\beta, \Lambda}(z)=\sum_{N \geq 0} \frac{z^{N}}{N !} \sum_{g \in \mathcal{G}_{N}} w_{\Lambda}(g), \quad \text { where } w_{\Lambda}(g):=\int_{\Lambda^{|g|}} \prod_{\{i, j\} \in E(g)} f_{i, j} \prod_{i=1}^{|g|} d q_{i},
$$

where by $|g|$ we denote the cardinality of the graph $g$ and we define it to be the number of vertices. Using the fact that the weight $w_{\Lambda}(g)$ is multiplicative on disconnected components a general theorem on enumeration of graphs gives (see e.g. [18] where it is stated as "The first Mayer Theorem")

$$
\sum_{N \geq 0} \frac{z^{N}}{N !} \sum_{g \in \mathcal{G}_{N}} w_{\Lambda}(g)=\exp \left\{\sum_{n \geq 1} \frac{z^{n}}{n !} \sum_{g \in \mathcal{C}_{n}} w_{\Lambda}(g)\right\},
$$

where $\mathcal{C}_{n}$ is the set of connected graphs on $n$ vertices. This is the predecessor of the Cluster Expansion Theorem 3.1! Then defining

$$
b_{n}(\Lambda):=\frac{1}{|\Lambda| n !} \sum_{g \in \mathcal{C}_{n}} w_{\Lambda}(g)
$$


(which is normalized in the volume and hence it has a limit $b_{n}:=\lim _{|\Lambda| \rightarrow \infty} b_{n}(\Lambda)$ ), equation (6.3) gives

$$
p_{\beta, \Lambda}(z)=\frac{1}{\beta|\Lambda|} \sum_{n \geq 1}|\Lambda| b_{n}(\Lambda) z^{n} \rightarrow \frac{1}{\beta} \sum_{n \geq 1} b_{n} z^{n} \equiv p_{\beta}(z) .
$$

In the thermodynamic limit the canonical free energy is the Legendre-Fenchel transform of the pressure, namely

$$
\beta f_{\beta}(\rho)=\sup _{z}\left\{\rho \log z-\beta p_{\beta}(z)\right\}=\rho \log z(\rho)-\beta p_{\beta}(z(\rho)),
$$

where $z(\rho)$ is given by the inversion of the relation $\rho=z p_{\beta}^{\prime}(z)$. Note that this is also equivalent to first defining the finite volume density by

$$
\rho_{\Lambda}(z):=\mathbb{E}_{G_{\beta, z, \Lambda}}[N]=z p_{\beta, \Lambda}^{\prime}(z)
$$

and then pass to the limit $|\Lambda| \rightarrow \infty$. In [18] this inversion is referred as "The second Mayer Theorem" and it is again a result on enumerating connected and 2-connected graphs, where the latter means all graphs which cannot be reduced to connected graphs by removing a point and all related edges. Under again the assumption that $w_{\Lambda}(g)$ is multiplicative (see in our case Lemma 5.2), we have that

$$
\left(\rho=z p_{\beta}^{\prime}(z)=\right) z \frac{\partial}{\partial z}\left(\sum_{n \geq 1} \frac{z^{n}}{n !} \sum_{g \in \mathcal{C}_{n}} w(g)\right)=z \exp \left\{\left.\frac{\partial}{\partial \rho}\left(\sum_{m \geq 2} \frac{\rho^{m}}{m !} \sum_{g \in \mathcal{B}_{m}} w(g)\right)\right|_{\rho: z=z(\rho)}\right\}
$$

where $\mathcal{B}_{m}$ is the set of 2 -connected graphs on $m$ vertices. Note that this is the combinatorial counterpart of our discussion in Section 5. From (6.12) we have that

$$
\rho=z p_{\beta}^{\prime}(z) \Leftrightarrow z(\rho)=\rho e^{-\sum_{m \geq 2} \beta_{m-1} \rho^{m-1}},
$$

where

$$
\beta_{m}:=\lim _{|\Lambda| \rightarrow \infty} \frac{1}{|\Lambda| m !} \sum_{g \in \mathcal{B}_{m+1}} w_{\Lambda}(g) .
$$

Plugging into $p_{\beta}(z(\rho))$ we obtain the famous virial expansion:

$$
\beta p_{\beta}(\rho)=\rho-\sum_{m \geq 1} \frac{m}{m+1} \beta_{m} \rho^{m+1} .
$$

Overall, (6.10), gives

$$
f_{\beta}(\rho)=\frac{1}{\beta}\left\{\rho(\log \rho-1)-\sum_{m \geq 1} \frac{1}{m+1} \beta_{m} \rho^{m+1}\right\} .
$$

\section{Acknowledgments.}

It is a great pleasure to thank Errico Presutti for suggesting us the problem discussed in this paper and for his continuous advising. We further acknowledge very fruitful discussions 
with Joel Lebowitz, Roman Kotecký, Marzio Cassandro, Enzo Olivieri, Suren Poghosyan, Daniel Ueltschi and Benedetto Scoppola. D.T. also acknowledges very kind hospitality of the Center for Theoretical Study at Prague and the Mathematics Institute of the University

of Warwick. The research of D.T. has been partially supported by a Marie Curie Intra European Fellowship within the 7th European Community Framework Program.

\section{REFERENCES}

[1] A. Bovier and M. Zahradník, A simple inductive approach to the problem of convergence of cluster expansion in polymer models, J. Stat. Phys. (2000), 100: 765-777.

[2] R. L. Dobrushin, Estimates of Semiinvariants for the Ising Model at Low Temperatures, Topics in Statistical Physics, AMS Translation Series 2, Vol. 177, AMS, Advances in the Mathematical Sciences-32. 1995, 59-81.

[3] R. Fernandez and A. Procacci: Cluster expansion for abstract polymer models. New bounds from an old approach, Comm. Math. Phys. (2007), 274, n.1, 123-140.

[4] R. Fernandez, A. Procacci, B. Scoppola, The Analyticity Region of the Hard Sphere Gas. Improved Bounds, J. Stat. Phys. (2007), 128: 1139-1143.

[5] M. Fischer, J. Lebowitz, Asymptotic Free Energy of a System with Periodic Boundary Conditions, Comm. Math. Phys. (1970), 19, 251-272

[6] C. Gruber and H. Kunz (1971), General properties of polymer systems, Comm. Math. Phys. (1971), 22, $133-61$.

[7] R. Kotecký and D. Preiss, Cluster expansion for abstract polymer models, Comm. Math. Phys., 103, 491-498, 1986.

[8] J. L. Lebowitz, A. Mazel and E. Presutti, Liquid-Vapor Phase Transitions for Systems with Finite-Range Interactions, J. Stat. Phys., 94, No. 5-6, 955-1025.

[9] J. L. Lebowitz and O. Penrose, Convergence of virial expansions, J. of Math. Phys. 5, 7, 1964.

[10] J. L. Lebowitz and O. Penrose, Rigorous Treatment of the Van Der Waals-Maxwell Theory of the Liquid-Vapor Transition, J. Math. Phys. 7, 98 (1966).

[11] P. Leroux, Enumerative problems inspired by Mayer's theory of cluster integrals, Electron. J. Combin. 11 (2004), no. 1.

[12] J. E. Mayer, Theory of Real Gases, Handbuch der Physik (Springer-Verlag, Berlin, 1958), Vol. 12.

[13] J. E. Mayer, M. G. Mayer, Statistical Mechanics, New York, John Wiley and Sons, 1940.

[14] F. R. Nardi, E. Olivieri and M. Zahradnik, On the Ising model with strongly anisotropic external field, J. Stat. Phys., Vol. 97, pag. 87-145 (1999).

[15] S. Poghosyan and D. Ueltschi, Abstract cluster expansion with applications to statistical mechanical systems, J. of Math. Phys. 50, 053509, 2009.

[16] D. Ruelle, Statistical Mechanics: rigorous results, World Scientific, Imperial College Press, 1969.

[17] A. Scott and A. Sokal, The Repulsive Lattice Gas, the Independent-Set Polynomial, and the Lovász Local Lemma, J. Stat. Phys. (2005), 118 (5-6): 1151-1261.

[18] G. E. Uhlenbeck and G. W. Ford, Lectures in Statistical Mechanics, AMS Providence, R. I., 1963.

[19] H. D. Ursell, The evaluation of Gibbs' phase-integral for imperfect gases, Proc. Camb. Phil. Soc. 23, 685 (1927). 
Elena Pulvirenti, Dipartimento di Matematica, Università di Roma 3

Rome, 00146, Italy

E-mail address: pulviren@mat.uniroma3.it

Dimitrios Tsagkarogiannis, Dipartimento di Matematica, Università di Roma Tor Vergata Rome, 00133, ITALY

E-mail address: tsagkaro@mat.uniroma2.it 